OPEN ACCESS

Edited by:

Sergey Brodsky,

Ohio State University Hospital,

United States

Reviewed by:

Shipra Agrawal,

Nationwide Children's Hospital,

United States

Wei Gong,

Nanjing Medical University, China

*Correspondence:

Yiming Zhou

zhouym35@mail.sysu.edu.cn

Anna Greka

agreka@bwh.harvard.edu

tThese authors have contributed equally to this work

${ }^{\ddagger}$ Present address: Yiming Zhou,

Medical Research Center, Sun Yat-sen Memorial Hospital, Sun Yat-sen University, Guangzhou, China

Specialty section:

This article was submitted to Nephrology,

a section of the journal

Frontiers in Medicine

Received: 07 June 2021

Accepted: 04 August 2021

Published: 21 September 2021

Citation:

Zhou Y, Kim C, Pablo JLB, Zhang F, Jung JY, Xiao L, Bazua-Valenti S, Emani $M$, Hopkins $C R$, Weins $A$ and

Greka A (2021) TRPC5 Channel

Inhibition Protects Podocytes in Puromycin-Aminonucleoside Induced Nephrosis Models.

Front. Med. 8:721865.

doi: 10.3389/fmed.2021.721865

\section{TRPC5 Channel Inhibition Protects Podocytes in Puromycin-Aminonucleoside Induced Nephrosis Models}

\author{
Yiming Zhou 1,2*f , Choah Kim ${ }^{2 \dagger}$, Juan Lorenzo B. Pablo ${ }^{2 \dagger}$, Fan Zhang ${ }^{1}$, Ji Yong Jung ${ }^{1,3}$, \\ Li Xiao ${ }^{1}$, Silvana Bazua-Valenti ${ }^{1,2}$, Maheswarareddy Emani ${ }^{2}$, Corey R. Hopkins ${ }^{4}$, \\ Astrid Weins ${ }^{5}$ and Anna Greka ${ }^{1,2 *}$

\begin{abstract}
${ }^{1}$ Department of Medicine, Brigham and Women's Hospital and Harvard Medical School, Boston, MA, United States, ${ }^{2}$ Center for the Development of Therapeutics (CDoT), Broad Institute of MIT and Harvard, Cambridge, MA, United States, ${ }^{3}$ Department of Internal Medicine, Gachon University Gil Medical Center, College of Medicine, Incheon, South Korea, ${ }^{4}$ Department of Pharmaceutical Sciences, University of Nebraska Medical Center, Omaha, NE, United States, ${ }^{5}$ Department of Pathology, Brigham and Women's Hospital and Harvard Medical School, Boston, MA, United States
\end{abstract}

Podocyte injury and the appearance of proteinuria are key features of several progressive kidney diseases. Genetic deletion or selective inhibition of TRPC5 channels with small-molecule inhibitors protects podocytes in rodent models of kidney disease, but less is known about the human relevance and translatability of TRPC5 inhibition. Here, we investigate the effect of TRPC5 inhibition in puromycin aminonucleoside (PAN)treated rats, human iPSC-derived podocytes, and kidney organoids. We first established that systemic administration of the TRPC5 inhibitor AC1903 was sufficient to protect podocyte cytoskeletal proteins and suppress proteinuria in PAN-induced nephrosis rats, an established model of podocyte injury. TRPC5 current was recorded in the human iPSC-derived podocytes and was blocked by AC1903. PAN treatment caused podocyte injury in human iPSC-derived podocytes and kidney organoids. Inhibition of TRPC5 channels reversed the effects of PAN-induced injury in human podocytes in both 2D and 3D culture systems. Taken together, these results revealed the relevance of TRPC5 channel inhibition in puromycin-aminonucleoside induced nephrosis models, highlighting the potential of this therapeutic strategy for patients.

Keywords: TRPC5 channel, calcium signaling, Rac1, podocyte, kidney disease

\section{INTRODUCTION}

Progressive chronic kidney disease (CKD) is associated with increased risk of kidney failure (1), and its prevalence is rapidly increasing with now more than 850 million people with CKD worldwide (2). Despite these rising numbers, the therapeutic options available to slow or prevent disease progression are limited $(3,4)$. Nephrotic syndrome is an important driver of CKD. Characterized by the presence of large amounts of albumin spilling into the urine, nephrotic syndrome is the consequence of damage to the filtering unit of the kidney, the glomerulus. When intact, the kidney filter, made up of endothelial cells, the basement membrane, and podocytes, is essential for retaining proteins in the blood and removing waste from the body. Many chronic kidney diseases are associated with the loss of podocytes, critical post-mitotic, terminally differentiated cells of the 
kidney filter that cannot be renewed once lost (5-9). Due to their limited capacity to proliferate, podocytes are especially vulnerable to various stimuli that lead to injury (10). Preventing podocyte injury therefore remains a critical objective for the development of effective, targeted therapeutic strategies for kidney diseases.

Numerous studies indicate that dysfunction of the podocyte cytoskeleton contributes to progressive proteinuric kidney diseases (3, 11), including Minimal Change Disease (MCD). Decreased expression of podocyte cytoskeletal proteins, including synaptopodin, nephrin, and podocin, is an early event in podocyte injury that results in the disorganization of the cytoskeleton, the fusion of foot processes, and ultimately the development of proteinuria and subsequent kidney damage (12). A significant number of mutations associated with filter barrier damage result in excess Rac1 signaling in podocytes including mutations in ARHGAP24 (13), ARHGDIA (14), and ARHGEF17 (15). A small GTP-binding protein, Rac1, is closely associated with various proteinuric kidney diseases, and critically, the regulation of podocyte cytoskeletal proteins. In addition to disruption of cytoskeletal protein remodeling, Rac1 activation results in increased ROS production and regulation of ion channels (16).

Ion channels are critical to kidney function, and their involvement in kidney disease is an active area of investigation. Transient receptor potential (TRP) channels are receptoroperated, non-selective, $\mathrm{Ca}^{2+}$-permeable, cationic channels that were first identified in Drosophila $(17,18)$. TRPC (TRP canonical) channels are a subgroup of this larger family that are particularly relevant to podocyte biology (19) and have been shown to play an important role in the pathogenesis of kidney disease. $\mathrm{Ca}^{2+}$ influx (20) through TRPC5 elicits dynamic and tightly regulated biochemical responses that activate Rac1. Rac1 activation leads to further vesicular insertion of TRPC5 into the plasma membrane, thus making more TRPC5 channels available for activation and completing a feed-forward pathway. Critically, data from three chemically distinct compounds that block TRPC5 activity (AC1903, ML204, and GFB-8438) have demonstrated beneficial effects when applied to rodent models of kidney disease (21). In addition to TRPC5, both gain-of-function $(22,23)$ and lossof-function mutations in TRPC6 channel activity contribute to podocyte injury (24), further implicating TRPC channel activity in chronic kidney diseases.

While the role of TRPC5 in podocyte injury has been defined using various rodent models, whether TRPC5 activity drives disease-relevant phenotypes in human kidney cells remains unexplored. The current study addresses these questions directly by harnessing the technological advances afforded by human induced pluripotent stem cell (iPSC)-derived 2D podocyte cultures (iPodos) and 3D kidney organoids. We determined that human podocytes express functional TRPC5 channels, and that TRPC5 inhibition protects human podocytes from injury. Our data were cross-validated in the experimentally tractable PAN-induced nephrotic rat model. This work provides a rationale for ongoing efforts to move TRPC5 inhibitors into the clinic (NCT03970122; https:// clinicaltrials.gov/) for the treatment of progressive proteinuric kidney diseases.

\section{RESULTS}

\section{Inhibition of TRPC5 Channel Activity Reduces Proteinuria and Protects Podocytes From Injury in PAN-Treated Rats}

Studies have shown that a single-dose of PAN administration to rats causes a marked nephrotic syndrome with severe proteinuria and the extent of damage depends on the amount and frequency of the PAN injection $(25,26)$. Many molecules, including TRPC6 channels, are associated with PAN-induced nephrosis in rats (2730 ). However, a recent study showed little to no protective effects in the early phase of PAN treatment in rats with genetic deletion of TRPC6 channels (31), suggesting that other pathways may mediate the early-stage disease. Previously, we have shown that inhibition of TRPC5 protects podocytes from injury and loss in the early phases of disease in several rodent models, suggesting a clinically relevant role for TRPC5 inhibition.

To investigate the role of TRPC5 in PAN nephrosis, we administered a single dose of PAN ( $50 \mathrm{mg} / \mathrm{kg}$ body weight rats), which induced a significant amount of urine albumin 7 days after injection. In contrast, co-administration of the TRPC5 channel inhibitor AC1903 twice per day significantly reduced urine albumin 7 days after PAN injection (Figure 1A). Periodic Acid Schiff (PAS) staining showed no obvious morphological changes in glomeruli and tubules from all groups (Figure 1B); however, transmission electron microscopy (TEM) showed extensive foot process effacement (FPE) without changes to the glomerular basement membrane (GBM) or the mesangial cells (Figure 1C), resembling the clinical manifestations of $\mathrm{MCD}$ in patients. Statistical analysis of rat podocyte foot processes (FPs) showed that treatment with AC1903 preserved FP number and protected FPs from effacement (Figures 1D,E). These results indicate that TRPC5 channels in vivo play an important role in inducing podocyte injury by PAN.

We further characterized the effects of PAN injection on several podocyte proteins. In PAN-induced nephrosis rats, the abundance of two podocyte cytoskeletal proteins, podocin and synaptopodin, was reduced in PAN-treated rat kidneys, while the expression levels of podocyte transcription factor WT1 were not affected, indicating that PAN at this concentration causes alterations in podocyte cytoskeletal structure but does not drive cell loss. Treatment with AC1903 successfully restored the PAN-induced depletion of podocin and synaptopodin (Supplementary Figure 1). Thus, we concluded that inhibition of TRPC5 channel activity can reduce FPE by protecting podocyte cytoskeletal structure.

\section{Systemic Administration of TRPC5 Inhibitor AC1903 Reduces PAN-Induced TRPC5 Activity}

To understand TRPC5 channel involvement and contribution to PAN-induced podocyte injury, we performed TRPC5 singlechannel recordings from acutely isolated rat kidney glomeruli according to our previously reported protocol and procedures (16). A single dose of PAN treatment successfully increased TRPC5 single-channel activity in response to the TRPC5 


\section{A}

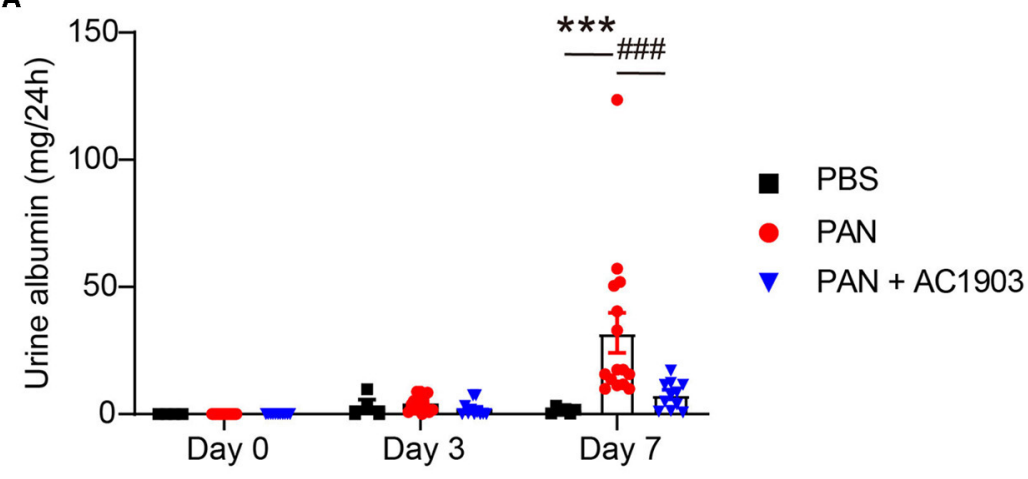

B

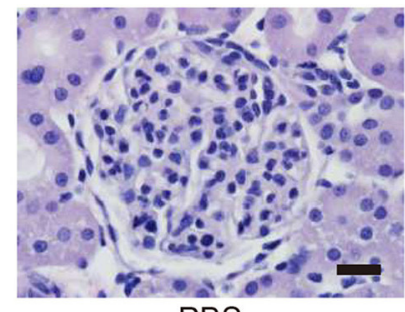

PBS

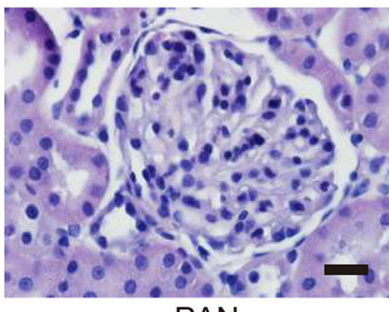

PAN

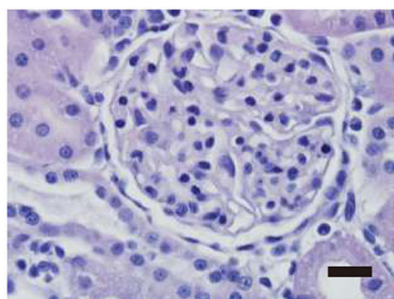

PAN + AC1903

C
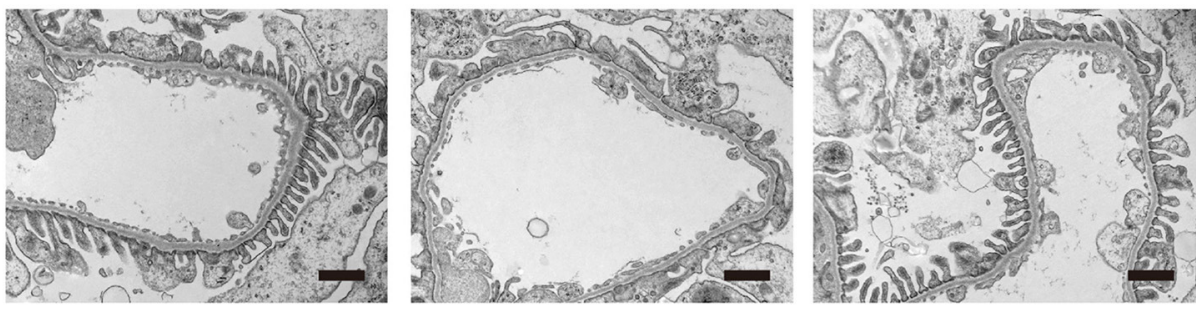

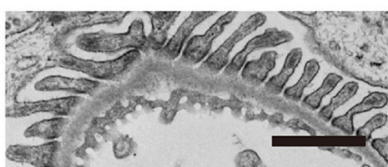

PBS

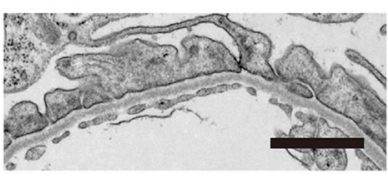

PAN

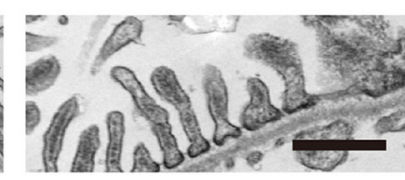

$\mathrm{PAN}+\mathrm{AC} 1903$
D

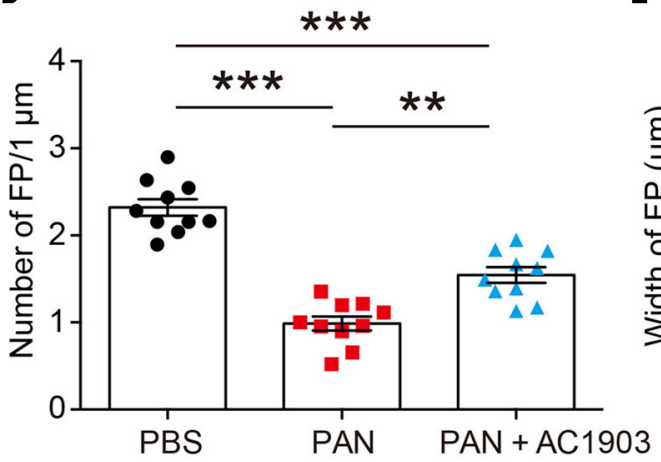

E

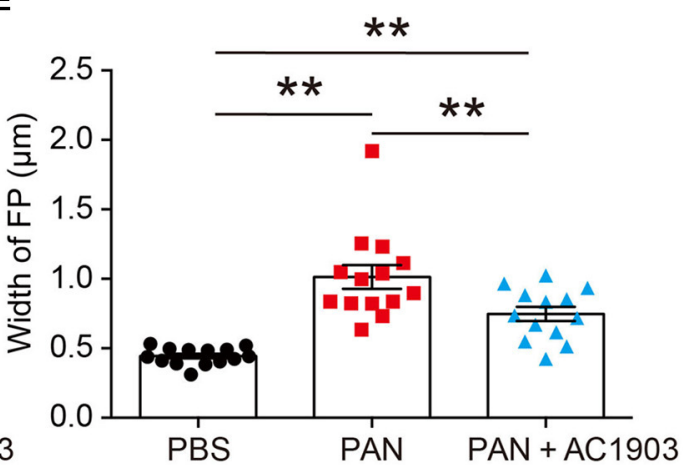

FIGURE 1 | AC1903 reduces proteinuria and protects podocytes from injury in a PAN nephrosis rat model. (A) 24-h urine albumin levels from PBS, PAN and PAN + AC1903 treated rats on day 0,3 and 7 . PAN $50 \mathrm{mg} / \mathrm{kg}$, AC1903 $50 \mathrm{mg} / \mathrm{kg}$. PBS $n=6 ;$ PAN $n=15 ;$ PAN + AC1903 $n=13 .{ }^{* * *} p<0.001$ PBS vs PAN, \#\#\# $p<$ 0.001 PAN vs PAN + AC1903. (B) Representative PAS staining images of PBS, PAN and PAN + AC1903 treated rats on day 7. Scale bar $20 \mu \mathrm{m}$. (C) Representative TEM images of podocyte foot processes (FPs) from PBS, PAN and PAN + AC1903 treated rat on day 7. Scale bar $1 \mu \mathrm{m}$. (D,E) Quantification of podocyte FPEs using the FP number (D) and width (E) on $1 \mu \mathrm{m}$ glomerular basement membrane from PBS, PAN and PAN + AC1903 treated rats on day $7 .{ }^{* \star} p<0.01,{ }^{\star \star \star} p<0.001$. 
agonist riluzole, while systemic co-administration of AC1903 with PAN in rats significantly reduced TRPC5 activity from isolated glomeruli (Figure 2A). PAN-treated rats showed a higher NPo value, the product of channel number and openchannel probability, while AC1903-treated rats exhibited a lower NPo value (Figure 2B). We hypothesized that systemic AC1903 administration would significantly lower the number of TRPC5 channels inserted in the podocyte plasma membrane, resulting in a low NPo. This result shows that, similar to observations in AT1R transgenic and Dahl spontaneous hypertensive rat models, AC1903 protects podocytes from PAN-induced injury when administered systemically by reducing TRPC5 channel activity.

\section{Human iPSC-Derived Podocytes Express Functional TRPC5 Channels}

Generating human podocytes in 2D cultures offers a unique opportunity to conduct mechanistic studies in vitro. To generate human iPodos, we adapted a previously published three-step protocol to induce differentiation into intermediate mesoderm, nephron progenitors, and finally, mature podocytes (32). iPodos exhibited typical in vitro mature podocyte morphology characterized by a large and flat cell body with a dense nucleus that resembled mouse and human immortalized podocytes $(33,34)$. The iPodos from this protocol expressed the major podocyte markers including SYNPO, NPHS1, NPHS2, and WT-1 (Supplementary Figure 2).

We performed patch clamp electrophysiology, the gold standard in measuring ion channel activity, using iPodos, 1214 days after induction. For whole-cell patch clamp recordings, a single iPodo was identified and the glass pipette was moved to the center of the cell body to provide a more effective Gigaseal (Figure 3A). Upon successful achievement of the whole-cell configuration, a strong outwardly rectifying current was observed upon application of a voltage ramp protocol, which decreased gradually within $30 \mathrm{~s}$ of perfusion (Figure 3B). Englerin A, a compound known to be a nanomolar activator of TRPC4 and TRPC5, was applied once the baseline became stable. Large outward and inward currents were induced by $100 \mathrm{nM}$ Englerin A, which could be blocked by TRPC5 inhibitor AC1903 (Figures 3B,C). The inhibitory effect of AC1903 was more prominent at negative potentials confirming that the baseline outwardly rectifying current did not correspond to a TRPC5 conductance (Figure 3D). These data provide the first evidence that human podocytes express functional TRPC5 channels at baseline, without additional manipulation, indicating that TRPC5 channels may play a role in human podocyte physiology.

Previous studies have indicated that TRPC5 activity is a major cause for podocyte injury in various rodent in vitro and in vivo models $(16,35)$. To determine whether inhibition of TRPC5 channel activity is protective in human podocytes, we investigated the effect of the TRPC5 channel inhibitor AC1903 on PAN-treated mature human iPodos. Our previous studies have demonstrated that activation of TRPC5 channels induces Rac1 activity in podocytes, which leads to the ROS production, cytoskeletal remodeling and podocyte loss in the angiotensin II type 1 receptor transgenic and spontaneous hypertensive nephropathy rat models (16). Therefore, we hypothesized that PAN treatment may cause iPodo injury through a similar mechanism. In support of this hypothesis, incubation with PAN for $24 \mathrm{~h}$ significantly increased iPodo intracellular ROS levels, which were reduced by co-treatment with AC1903 (Figures 3E,F). These results suggest that inhibition of TRPC5 channels by the small molecule AC1903 can protect human iPodos from PAN-induced ROS generation. Previous experiments in mouse podocytes have shown that AC1903 blocks ROS generation induced by angiotensin II (AngII), suggesting that both mouse immortalized podocytes, and now human iPodos, support a role for TRPC5 in podocyte biology and disease pathophysiology. In summary, using iPodos, we demonstrated the presence of active TRPC5 channels, inhibition with AC1903, and measured downstream reduction of ROS, the sequela of PAN-mediated TRPC5 activation.

\section{TRPC5 Inhibition Preserves Podocin, Synaptopodin, and Nephrin Abundance in PAN-Treated Human Kidney Organoids}

To further evaluate the effect of AC1903 in podocytes as well as other kidney cells, we took advantage of the human iPSC-derived kidney organoid model. Organoids contain selforganized nephrons composed of early glomerular structures connected to tubular cells including proximal tubules, loops of Henle and distal tubules. These 3D organoids thus hold the potential to be excellent in vitro models for preclinical drug testing, because they allow simultaneous monitoring of drug effects on multiple kidney cell types (36-38). We differentiated kidney organoids for 25 days and observed nephron-like structures that were similar with previous reports (Supplementary Figure 3). Using immunofluorescence imaging, we found that PAN treatment reduced nephrin, podocin and synaptopodin, but not WT1 expression levels (Figure 4; Supplementary Figure 4). Co-treatment with AC1903 preserved podocyte cytoskeletal proteins, as also observed in PAN rats. Taken together, our data suggest that inhibition of TRPC5 channel activity protects human podocytes from PAN-induced injury in an in vitro 3D model.

\section{DISCUSSION}

The majority of studies investigating podocyte biology in vitro have relied on immortalized cell lines. Although these cell lines express podocyte markers such as podocin, synaptopodin, nephrin, and WT-1, and respond to many stimuli, they are prone to de-differentiation, resulting in variability. Moreover, immortalized cell lines fall short of recapitulating the crosstalk and interactions between podocytes and other cell types of the kidney. Following successful generation of 2D human podocyte cultures (iPodos) and 3D kidney organoids in vitro from human iPSCs, we showed that PAN treatment causes elevated ROS in iPodos and disruption of podocyte cytoskeletal proteins, such as synaptopodin and podocin in kidney organoids, through the activation of TRPC5. We demonstrated the podocyte-protective effects of small-molecule inhibitors of TRPC5 channels in human 
A

\section{Riluzole}

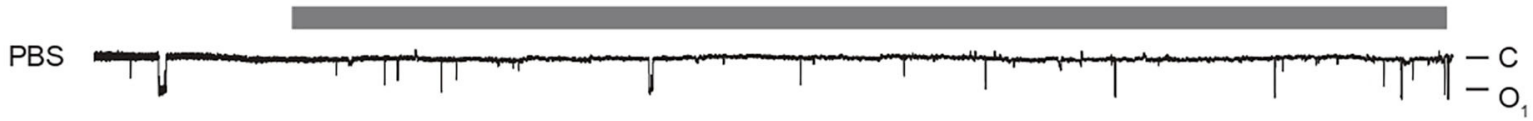

Riluzole

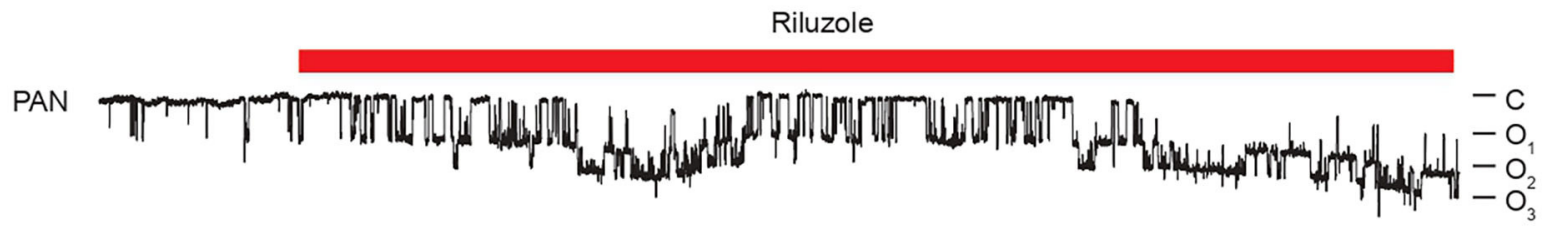

Riluzole

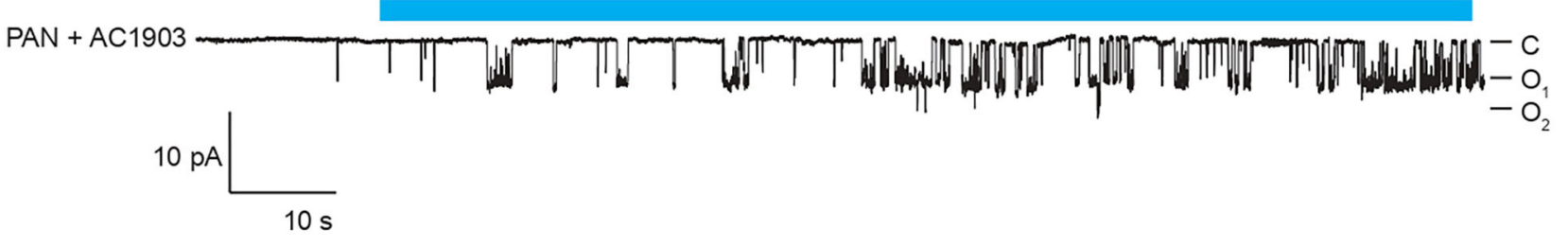

B

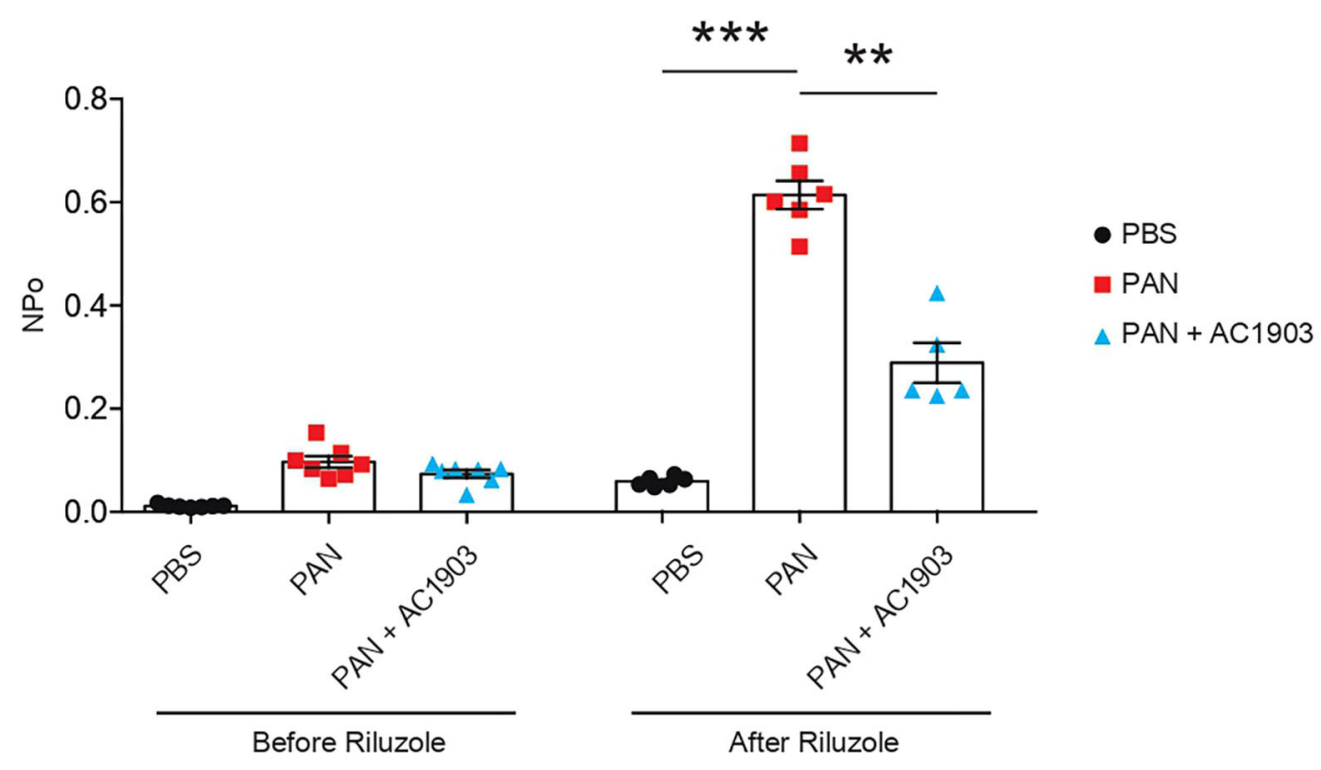

FIGURE 2 | Single-channel recordings from acutely isolated glomeruli show that systemic treatment with AC1903 abrogates TRPC5 activity. (A) Representative TRPC5 single-channel current traces from PBS, PAN and PAN + AC1903 treated rats in response to TRPC5 channel agonist Riluzole (30 $\mu$ M). (B) Quantification of TRPC5 single-channel activity by analysis of NPo values. ${ }^{\star \star} p<0.01,{ }^{\star \star \star} p<0.001$.

podocytes and kidney organoids, establishing these systems as reproducible, human-specific tools to study podocyte-associated kidney diseases $(32,36-38)$.

Our previous data demonstrated TRPC5 expression and activity in mouse kidney tissues as well as rat glomeruli, and that a TRPC5-specific small-molecule inhibitor AC1903, can rescue podocytes and attenuate the progression of kidney diseases in angiotensin II type 1 receptor transgenic, and spontaneous hypertensive rat models $(16,35)$. Mechanistically, activation of TRPC5 channels by angiotensin II type 1 receptor increases Racl activity, which increases ROS production and cytoskeletal remodeling in podocytes, eventually leading to podocyte injury and loss. These findings provided a mechanistic rationale for therapeutically targeting TRPC5 channels in the 
A

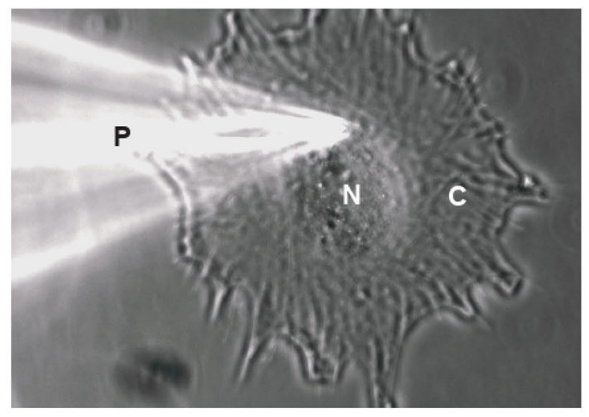

C

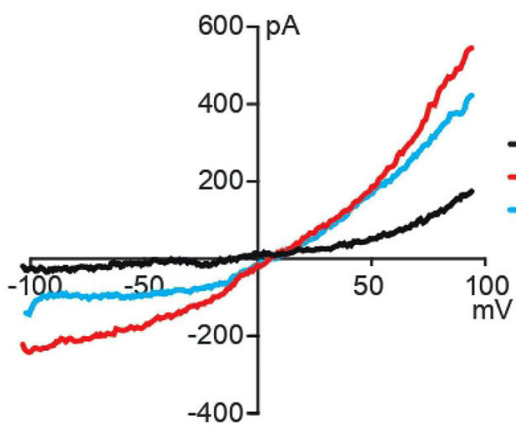

B

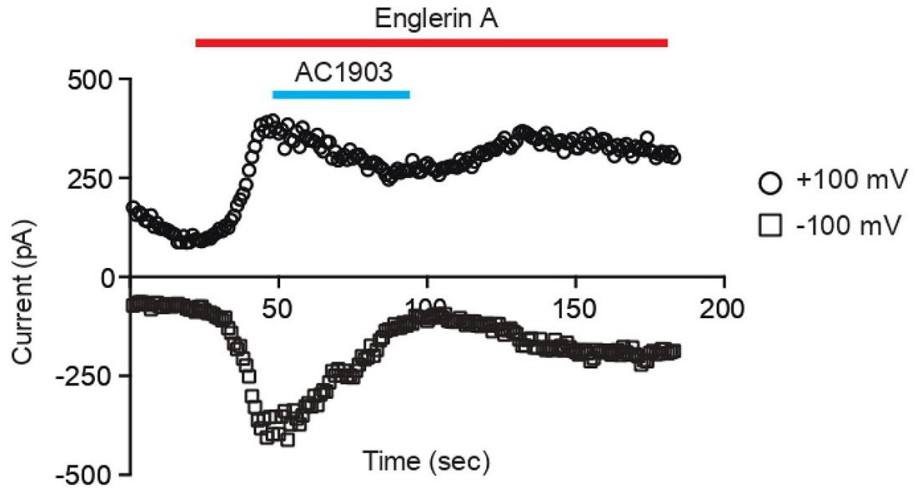

D

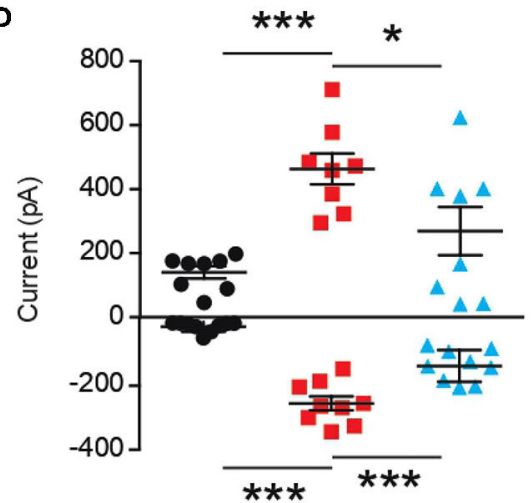

Baseline Englerin $\mathrm{A}$

Englerin A + AC1903

E

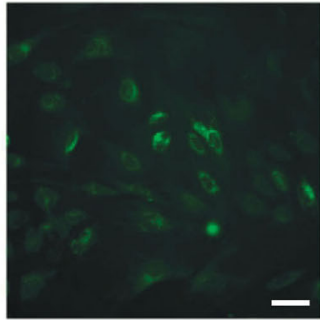

PBS

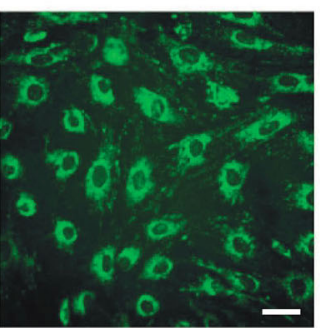

PAN

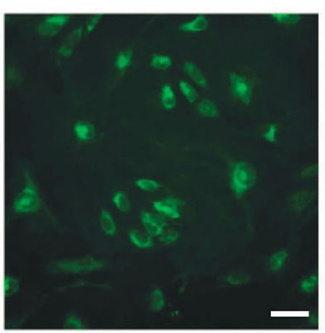

$\mathrm{PAN}+\mathrm{AC} 1903$

$\mathbf{F}$

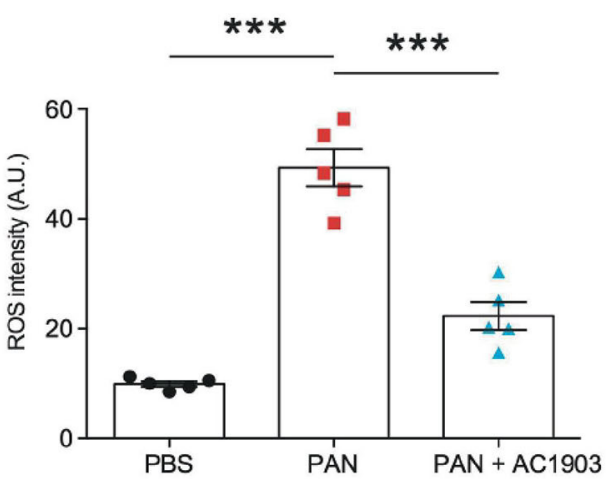

FIGURE 3 | Functional TRPC5 channel activity blocked by AC1903 reduces cytosolic ROS and protects cytoskeletal proteins in PAN-treated iPodos. (A) Representative image of a human iPodo patch clamp recording in the whole-cell configuration. P, Glass pipette; N, iPodo nuclear; C, iPodo cytosol. (B) Representative 
FIGURE 3 | diary plots of whole-cell currents from iPodos in response to TRPC5 channel agonist Englerin A (100 nM) in the absence or presence of TRPC5 channel inhibitor AC1903 (30 $\mu \mathrm{M}$ ). Currents shown are from $+100 \mathrm{mV}$ and $-100 \mathrm{mV}$ of a ramp protocol. (C) Representative TRPC5 channel current-voltage (I-V) curves from iPodo whole-cell recording. (D) Statistical analysis of I-V curves from iPodos treated with Englerin $A$ in the absence or presence of $A C 1903 .{ }^{*} p<0.05$, ${ }^{\star \star \star} p<0.001$. (E) Representative cytosolic ROS images in iPodos after 24-h treatment with PAN (150 $\mu \mathrm{g} / \mathrm{mL})$ with or without AC1903 (30 $\mu$ M). Scale bar $10 \mu \mathrm{m}$. (F) Statistical analysis of the ROS signal intensities. ${ }^{* \star} p<0.001$.

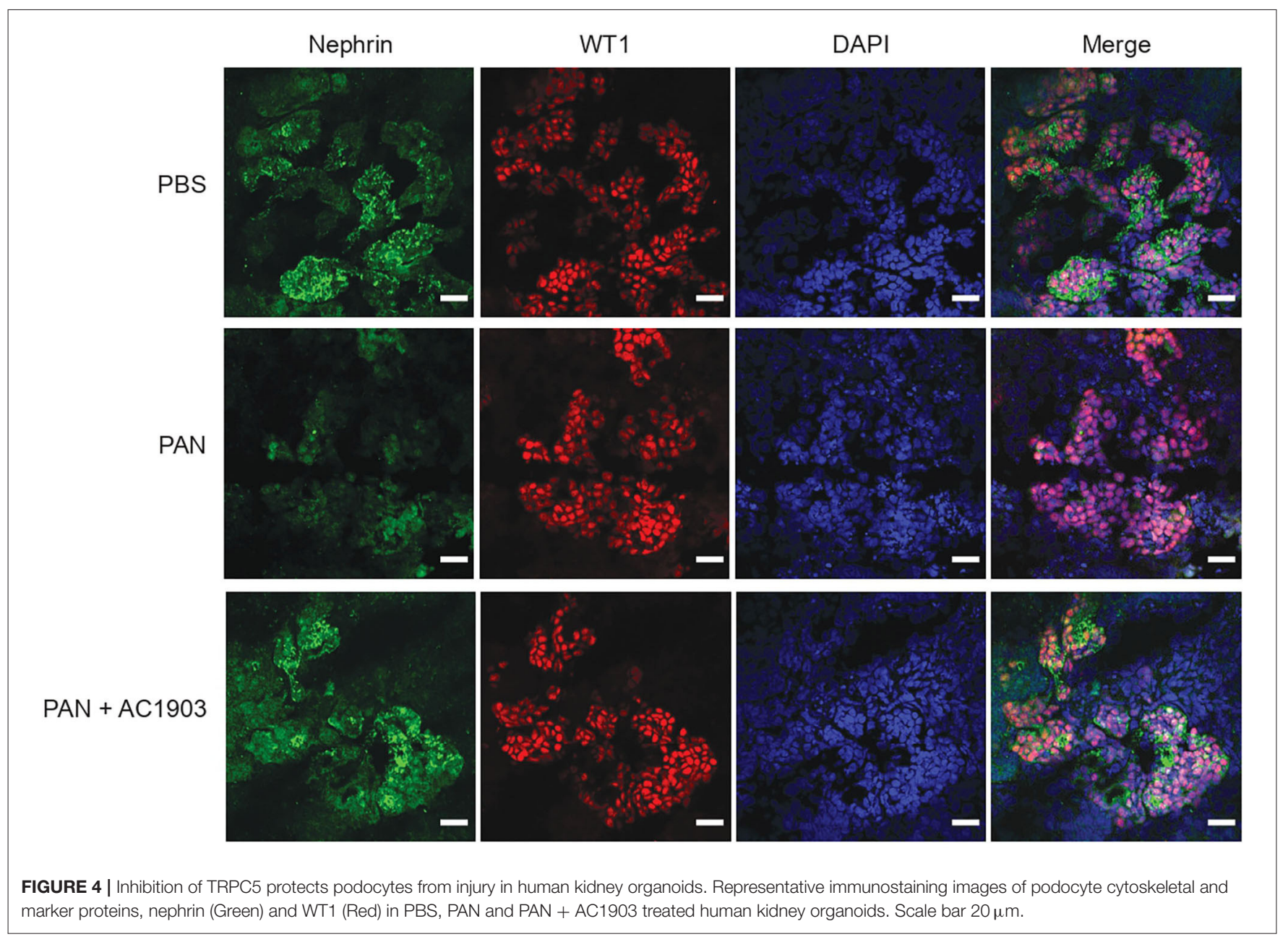

treatment of progressive chronic kidney diseases. In this study, we generated human iPSC-derived podocytes and used patch clamp electrophysiology to demonstrate their response to a more potent TRPC $4 / 5$ channel agonist, Englerin A. The currents induced by Englerin A were blocked by the TRPC5-selective inhibitor AC1903. We believe these results further strengthen the idea that AC1903 remains effective with different TRPC5 channel activators. To our knowledge, this is the first demonstration that human podocytes express functional TRPC5 channels, further strengthening the notion that these channels play an important role in progressive kidney diseases.

Prior work has shown that Racl activation is a nodal event in a spectrum of glomerular diseases, while inhibition of Rac1 activity ameliorates podocyte injury in response to various noxious stimuli $(14,39-42)$. In this study, we investigated the contribution of the TRPC5 channel in a PAN-induced nephrosis rat model. In contrast to angiotensin II type 1 receptor transgenic and spontaneous hypertensive rat models, PANinduced nephrosis rats displayed strong foot process effacement, but not podocyte loss, resembling the clinical phenotype of MCD. A single dose of PAN was sufficient to induce podocyte injury and proteinuria in rats within a week, which was consistent with the PAN-induced injury observed in human podocytes and kidney organoids. Previously, we confirmed the protective effect of AC1903 both before and after disease onset in two rodent models (16). In this study, therefore, we decided to administer AC1903 before disease onset, since our goal was to test whether inhibition of TRPC 5 can prevent podocyte injury at an early time point. As expected, inhibition of TRPC 5 channels by AC1903 was sufficient to protect podocyte cytoskeletal proteins and suppress proteinuria in PAN-induced nephrosis rats within a week. The accelerated timeline of injury in PAN-induced 
nephrosis increases the ease with which this model could be used in pre-clinical studies, especially in comparison to AT1R transgenic and Dah1 Salt-sensitive spontaneous hypertensive rat models which require at least a month for podocyte injury and proteinuria to be established. A recent study has also shown that another TRPC5 channel inhibitor GFB-8438, which has a $\mathrm{IC}_{50}$ of $0.18 \mu \mathrm{M}$, protects mouse podocytes from injury induced by protamine sulfate (PS) in vitro and significantly reduces both urinal total protein and albumin levels in a hypertensive deoxycorticosterone acetate (DOCA)-salt rat model of FSGS (43). We speculate that the effects of AC1903 and GFB-8438 would be comparable in the PAN rat model as well.

Taken together, these data indicate that inhibition of TRPC5 channel activity protects podocytes from PAN-induced injury. Our data also highlight the utility of human iPodos, kidney organoids, and the PAN-induced nephrosis rat model as useful tools for the preclinical development of TRPC5 channel inhibitors. This diverse set of validated models spans both human in vitro systems conducive for mechanistic studies and experimentally tractable in vivo disease models with physiological readouts. In sum, this study bolsters the human relevance and scientific rationale for a TRPC5-targeted podocyteprotective strategy.

\section{METHODS}

\section{Animals}

Wild-type Sprague-Dawley rats (Male, 4-5 weeks, Charles River) were housed under a controlled environment with a $12 \mathrm{~h}$ light-dark cycle and access to food and water ad libitum. All animal experiments were performed in accordance with the guidelines established and approved by the Animal Care and Use Committee at Brigham and Women's Hospital, Harvard Medical School (2016N00146). After wild-type Sprague-Dawley rats were acclimated for a week in the BWH CCM animal facility. A single dose of puromycin aminonucleoside (50 mg/kg, PAN group) was given i.p. to rats to induce nephrosis, and PBS was given as control. Following the PAN injection, vehicle or AC1903 (50 $\mathrm{mg} / \mathrm{kg}$ ) was administered twice daily (at 9 am and $9 \mathrm{pm}$ ) for seven days. Twenty-four-hour urine albumin levels were measured on day 0,3 , and 7 . Rats were euthanized after the metabolic collection on day 8 . Both kidneys were collected for downstream experiments. In most cases, one kidney was used for acute glomeruli isolation and glomerular single-channel recording. For immunostaining, another kidney was quickly removed and cut into half. One half was flash frozen in liquid nitrogen, and the other was fixed in 4\% PFA overnight and stored in PBS for follow-up experiments. In combination, we have studied 34 rats (PBS group $n=6$, PAN group $n=15$, PAN $+\mathrm{AC} 1903$ group $n=13$ ).

\section{Chemical Preparation and IP Administration}

All chemicals were purchased from Sigma-Aldrich unless described otherwise. AC1903 was synthesized, purified and prepared by C.H. as previously published (16). (-) Englerin A was purchased from Phytolab (\#82530). Immediately prior to injections, AC1903 solution was placed on a heated shaker at $48^{\circ} \mathrm{C}$ and $800 \mathrm{rpm}$. Vehicle was prepared in the same fashion. Injection amount was determined by body weight ( $2 \mathrm{~mL}$ vehicle/compound per $\mathrm{kg}$ body weight). Body weight was measured at the time of injection.

\section{Metabolic Collection and Urine Albumin Assay}

Rats were housed individually in a metabolic cage supplied with adequate amounts of food and water. Urine was collected into a $50 \mathrm{~mL}$ Falcon tube for $24 \mathrm{~h}$. Total urine volume was measured and then centrifuged at $3,200 \times \mathrm{g}$ for $10 \mathrm{~min}$ at $4^{\circ} \mathrm{C}$. Albumin quantification was done according to our previously published protocol (16). Coomassie Brilliant Blue stained gels of urine samples were quantified by densitometry with albumin standards using Image J software.

\section{Human iPSC Culture}

Human Episomal iPSC Line (ThF) (ThermoFisher, \#A18945) was maintained in mTeSR1 medium (Stem Cell Technologies, \#85870) in T25 flasks pre-coated with Matrigel (Stem Cell Technologies, \#354277). Cells were passaged using Gentle Cell Dissociation Reagent (Stem Cell Technologies, \#7174). iPSCs were confirmed to be karyotype normal and maintained below passage 10 and all the cell lines were routinely checked and were negative for mycoplasma.

\section{Differentiation Into Human iPSC-Derived Podocytes}

Human iPSC-derived podocytes (iPodos) were generated using the cited protocol with a few modifications (32). A total number of $3.75 \times 10^{5} \mathrm{ThF}$ human iPSCs were seeded in a Matrigel-coated T25 flask in mTeSR1 medium (Stem Cell Technologies, \#85870) with ROCK inhibitor, Y-27632 (10 $\mu \mathrm{M}$, Stem cell Technologies, \#72304). After $24 \mathrm{~h}$ cells were treated with a 1:1 mixture of DMEM/F12 + GlutaMAX (Life Technologies, \#10565-018) and Neurobasal media, supplemented with N2 and B27 (Life Technologies, \#21103049), CP21R7 (1 $\mu \mathrm{M}$, Cayman Chemical, \#20573), and BMP4 (25 ng/mL, Peprotech, \#AF-120-05ET), for 3 days. On day four, the medium was replaced with STEMdiff APEL2 medium (Stem Cell Technologies, \#05270) supplemented with FGF9 (200 ng/mL, Peprotech, \#100-23), BMP7 (50 ng/mL, Peprotech, \#120-03), and Retinoic Acid (100 nM, Sigma-Aldrich, \#R2625) for 2 days. On day six, cells were dissociated with Accutase (Stem Cell Technologies, \#07920) and $2 \times 10^{5}$ cells were seeded on Type I Collagen-coated 6-well dishes and cultured until day fourteen in DMEM/F12+GlutaMAX medium supplemented with 10\% FBS (Life Technologies, \#16140071). Vitamin D3 (100 nM, Tocris Bioscience, \#4156), and Retinoic Acid (100 $\mu \mathrm{M}$, Sigma-Aldrich, \#R2625) were added every other day. Cells were fully differentiated and ready to use from Day 12 to Day 14.

\section{Kidney Organoid Differentiation}

Kidney organoids were generated using a previously described protocol (37) with slight modifications. A total number of $3.75 \times$ $10^{5} \mathrm{ThF}$ iPSCs were plated in a T25 flask in the mTeSR 1 medium 
with ROCK Inhibitor Y-27632 $(10 \mu \mathrm{M}$, Stem cell Technologies, \#72304). After $24 \mathrm{~h}$, cells were treated with CHIR99021 (8 $\mu \mathrm{M}$, R\&D systems, \#4423/10) in the STEMdiff APEL2 medium (Stem Cell Technologies, \#05270) for 4 days, followed by recombinant human FGF-9 (200 ng/mL, Peprotech, \#100-23) and heparin $(1 \mu \mathrm{g} / \mathrm{mL}$, Sigma-Aldrich, \#H4784) for an additional 3 days. At day seven, cells were dissociated into single cells using AccutaseTM (Stem Cell Technologies, \#07920). $5 \times 10^{5}$ cells were pelleted at $350 \mathrm{x} g$ for $2 \mathrm{~min}$ and transferred onto a 6-well transwell membrane (Stem Cell Technologies, \#3450). Pellets were incubated with CHIR99021 $(5 \mu \mathrm{M})$ in the APEL2 medium for $1 \mathrm{~h}$ at $37^{\circ} \mathrm{C}$. Then the medium was changed to the APEL2 medium with FGF-9 $(200 \mathrm{ng} / \mathrm{mL})$ and heparin $(1 \mu \mathrm{g} / \mathrm{mL})$ for an additional 5 days, and an additional 2 days with heparin $(1 \mu \mathrm{g} / \mathrm{mL})$. Medium was changed every other day. The organoids were maintained in APEL2 medium with no additional factors until day 25. Then kidney organoids were treated with PBS, PAN $(150 \mu \mathrm{g} / \mathrm{mL})$ with or without AC1903 $(30 \mu \mathrm{M})$ for $72 \mathrm{~h}$ before the downstream experiments.

\section{ROS Assay}

Human iPSC-derived podocytes (iPodos) were treated with either PBS, PAN $(150 \mu \mathrm{g} / \mathrm{mL})$, or PAN with $30 \mu \mathrm{M}$ AC1903 for $24 \mathrm{~h}$. Intracellular production of ROS was measured using a cell-permeable fluorescent ROS indicator (Invitrogen, \#C10444) following the official protocol. Briefly, cells were incubated with $5 \mu \mathrm{M}$ CellRox Green at $37^{\circ} \mathrm{C}$ for $30 \mathrm{~min}$ in Hanks' balanced salt solution (ThermoFisher Scientific, \#14025092). Cells were then washed with PBS and fixed with $4 \%$ PFA. Fluorescence images were taken under a confocal microscope Olympus FV-1000. Each cell was circled and the ROS signal intensities were measured using ImageJ software. Average ROS intensities were normalized with their cell number in each group.

\section{Rat Kidney Immunofluorescence}

For immunofluorescence, kidney tissues were sectioned at $6 \mu \mathrm{m}$ thickness and blocked with $3 \%$ BSA at room temperature for $1 \mathrm{~h}$. The rabbit anti podocin, guinea pig anti synpo, goat anti Nephrin, and rabbit anti WT-1 antibodies were used at a dilution of 1:200. The Alexa goat anti rabbit and guinea pig IgG 488 and Alexa donkey anti goat IgG 594 were used at a dilution of 1:200. Fluorescence images were taken with a confocal microscope Olympus FV-1000.

\section{Periodic Acid Schiff Staining}

Paraffin-embedded rat kidney slides were deparaffinized and hydrated with distilled water. Then they were oxidized in 0.5\% Periodic Acid solution for $5 \mathrm{~min}$, and rinsed 3 times with distilled water. Slides were then placed in Schiff's reagent for $15 \mathrm{~min}$ and washed with tap water for $5 \mathrm{~min}$. Slides were counterstained in Mayer's hematoxylin for $1 \mathrm{~min}$ and washed with tap water for $5 \mathrm{~min}$ and then rinsed with distilled water. Slides were finally dehydrated and mounted using Xylene based mounting media.

\section{Transmission Electron Microscopy and Podocyte Foot Process Quantification}

Kidney samples were fixed in $3 \%$ glutaraldehyde and $2 \%$ paraformaldehyde in $0.1 \mathrm{M}$ cacodylate buffer $(\mathrm{pH} 7.3)$ at $4^{\circ} \mathrm{C}$ overnight. On the second day, samples were washed in $0.1 \mathrm{M}$ sodium cacodylate buffer and then post-fixed in $1 \%$ osmium tetroxide for $1 \mathrm{~h}$. Samples were dehydrated with alcohol, and then embedded in Epon resin (Electron Microscopy Science). Sections (60-100 nm) were prepared with an EM UC7 ultramicrotome (Leica Microsystems, Germany), and stained with uranyl acetate and lead citrate. The stained samples were examined in a JEM 2100 transmission electron microscope (JEOL, USA). The quantification of podocyte foot process was performed with ImageJ software. First of all, lines were drawn along the GBM or across each foot process, and then the length of GBM and the width of each foot process were measured with ImageJ software. The average number and width of foot processes per micrometer length of GBM were calculated for the statistical analysis.

\section{Electrophysiology}

iPodo whole-cell patch clamp was performed using an Axopatch 700B and Digidata 1550A (Molecular Devices). Bath solution contained (in mM) $135 \mathrm{CH}_{3} \mathrm{SO}_{3} \mathrm{Na}, 5 \mathrm{CsCl}, 2 \mathrm{CaCl}_{2}, 1 \mathrm{MgCl}_{2}, 10$ HEPES, and 10 glucose adjusted with $\mathrm{NaOH}$ to $\mathrm{pH}$ 7.4. Pipette solution contained (in $\mathrm{mM}$ ) $135 \mathrm{CH}_{3} \mathrm{SO}_{3} \mathrm{Cs}, 10 \mathrm{CsCl}, 3 \mathrm{MgATP}$, 0.2 EGTA, $0.13 \mathrm{CaCl}_{2}$, and 10 HEPES adjusted with $\mathrm{CsOH}$ to $\mathrm{pH}$ 7.4. For glomerular single-channel recording, acutely isolated glomeruli were prepared as previously published (16). Singlechannel recordings were carried out using an Axopatch 200B and Digidata 1550A (Molecular Devices). Bath and pipette solutions for glomerular single-channel recording contained (in $\mathrm{mM}$ ) 135 $\mathrm{CH}_{3} \mathrm{SO}_{3} \mathrm{Na}, 5 \mathrm{CsCl}, 2 \mathrm{CaCl}_{2}, 1 \mathrm{MgCl}_{2}, 10 \mathrm{HEPES}$, and 10 glucose adjusted with $\mathrm{NaOH}$ to $\mathrm{pH}$ 7.4. Once the inside-out configuration was achieved, the bath solution was replaced by an intracellular solution, containing (in $\mathrm{mM}$ ) $135 \mathrm{CH}_{3} \mathrm{SO}_{3} \mathrm{Cs}$, $10 \mathrm{CsCl}, 3 \mathrm{MgATP}, 0.2 \mathrm{EGTA}, 0.13 \mathrm{CaCl}_{2}$, and $10 \mathrm{HEPES}$ adjusted with $\mathrm{CsOH}$ to $\mathrm{pH}$ 7.4. Patch pipettes, with a resistance of 4-6 M $\Omega$, were prepared using a two step-protocol (Sutter Instrument, P-97). Pipettes were fire-polished before use with a microforge (Narishige, MF-9). For glomerular single-channel recording, data was acquired at $10 \mathrm{kHz}$ sampling frequency, and filtered with low-pass filtering at $1 \mathrm{kHz}$. Holding membrane potential was at $-60 \mathrm{mV}$. Single-channel analysis was carried out using Clampfit 10.4 software (Molecular Devices). NPo were analyzed for $10 \mathrm{~s}$ before and after the application of TRPC5 agonist riluzole (Sigma, R116).

\section{Statistical Analysis}

All the data were presented as Mean \pm SEM unless described otherwise. Microsoft Office Excel, Origin 6.0 and Graphpad Prism 6 were used for statistical analysis and creation of the graphs. For statistical analysis of differences, an unpaired $t$ test and a one-way ANOVA followed by Bonferroni or Tukey Correction were used. $p$-value $<0.05$ was considered to be significant. 


\section{DATA AVAILABILITY STATEMENT}

The original contributions presented in the study are included in the article/Supplementary Material, further inquiries can be directed to the corresponding author/s.

\section{ETHICS STATEMENT}

The animal study was reviewed and approved by the Animal Care and Use Committee at Brigham and Women's Hospital, Harvard Medical School.

\section{AUTHOR CONTRIBUTIONS}

$\mathrm{YZ}$ and AG designed the experiments. YZ, FZ, JJ, and LX performed animal experiments. YZ, CK, SB-V, and JP performed iPodocyte electrophysiology. YZ performed iPodocyte ROS assay. ME performed kidney organoid experiments. AW and $\mathrm{YZ}$ quantified the histology. $\mathrm{YZ}, \mathrm{CH}$, and $A G$ prepared the figures and wrote the manuscript. All authors contributed to the article and approved the submitted version.

\section{FUNDING}

This work was supported by NIH/NIDDK grants DK099465 and DK095045 (AG).

\section{REFERENCES}

1. Glassock RJ, Warnock DG, Delanaye P. The global burden of chronic kidney disease: estimates, variability and pitfalls. Nat Rev Nephrol. (2017) 13:10414. doi: $10.1038 /$ nrneph.2016.163

2. Jager KJ, Kovesdy C, Langham R, Rosenberg M, Jha V, Zoccali C. A single number for advocacy and communication-worldwide more than 850 million individuals have kidney diseases. Nephrol Dial Transplant. (2019) 34:18035. doi: 10.1093/ndt/gfz174

3. D'agati VD. Pathobiology of focal segmental glomerulosclerosis: new developments. Curr Opin Nephrol Hypertens. (2012) 21:243-50. doi: 10.1097/MNH.0b013e32835200df

4. Jha V, Garcia-Garcia G, Iseki K, Li Z, Naicker S, Plattner B, et al. Chronic kidney disease: global dimension and perspectives. Lancet. (2013) 382:26072. doi: 10.1016/S0140-6736(13)60687-X

5. D’agati VD, Kaskel FJ, Falk RJ. Focal segmental glomerulosclerosis. $N$ Engl J Med. (2011) 365:2398-411. doi: 10.1056/NEJMra1106556

6. Brinkkoetter PT, Ising C, Benzing T. The role of the podocyte in albumin filtration. Nat Rev Nephrol. (2013) 9:328-36. doi: 10.1038/nrneph.2013.78

7. Jefferson JA, Shankland SJ. The pathogenesis of focal segmental glomerulosclerosis. Adv Chronic Kidney Dis. (2014) 21:40816. doi: 10.1053/j.ackd.2014.05.009

8. Noone DG, Iijima K, Parekh R. Idiopathic nephrotic syndrome in children. Lancet. (2018) 392:61-74. doi: 10.1016/S0140-6736(18)30536-1

9. Yoshimura Y, Nishinakamura R. Podocyte development, disease, and stem cell research. Kidney Int. (2019) 96:1077-82. doi: 10.1016/j.kint.2019.04.044

10. Wiggins RC. The spectrum of podocytopathies: a unifying view of glomerular diseases. Kidney Int. (2007) 71:1205-14. doi: 10.1038/sj.ki.5002222

11. Greka A, Mundel P. Cell biology and pathology of podocytes. Annu Rev Physiol. (2012) 74:299-323. doi: 10.1146/annurev-physiol-020911-153238

\section{SUPPLEMENTARY MATERIAL}

The Supplementary Material for this article can be found online at: https://www.frontiersin.org/articles/10.3389/fmed. 2021.721865/full\#supplementary-material

Supplementary Methods | Total mRNA was extracted from $5 \times 10^{6}$ hiPSC and iPodo cells with TRIzol reagent according to the official protocol (ThermoFisher Scientific, USA). The cDNA was synthesized by ReverTra Ace kit (Toyobo, Japan). qPCR was then performed using a SYBR Green qPCR Kit (ThermoFisher Scientific, US) with the CFX Real-Time PCR system (Bio-Rad, USA). GAPDH (Forward 5'-GTCTCCTCTGACTTCAACAGCG-3', Reverse 5'-ACCACCCTGTTGC TGTAGCCAA-3'); SYNPO (Forward 5'-TCCTTCATGTTGCTGCCGAT-3', Reverse 5'-AGATCCTTCTCCGTGAGGCT-3'); NPHS2 (Forward 5'-ACCAAATCCTCCGGC TTAGG-3', Reverse 5'-CAACCTTTACGCAGAACCAGA-3'); NPHS1 (Forward 5'-GTCTGCACTGTCGATGCCAATC-3', Reverse 5'-CCAGTTTGGCATGGTGAAT CCG-3'); WT1 (Forward 5'-CGAGAGCGATAACCACACAACG-3', Reverse 5'-GTC TCAGATGCCGACCGTACAA-3').

Supplementary Figure 1 | Inhibition of TRPC5 channel activity protects podocytes in PAN rats. (A) Representative immunostaining images of podocyte cytoskeletal and marker proteins podocin, synaptopodin, and WT1 from PBS, PAN and PAN + AC1903 treated rats on day 7. Scale bar $20 \mu \mathrm{m}$.

Supplementary Figure 2 | Expression levels of SYNPO, NPHS1, NPHS2, and WT1 in human iPSC-derived podocytes. (A-D) qPCR results of podocyte markers SYNPO (A), NPHS1 (B), NPHS2 (C), and WT1 (D) from hiPSC and iPodo. hiPSC $n=3$, iPodo $n=3$. ${ }^{* *} p<0.01$ vs. hiPSC.

Supplementary Figure $\mathbf{3}$ | Bright-field images of human iPSCs and kidney organoids. (A) Bright-field images of human iPSCs (A, scale bar $200 \mu \mathrm{m}$ ), kidney organoid at day 6 (B), day 15 (C), and day 25 (D). Scale bars (B-D) $50 \mu \mathrm{m}$.

Supplementary Figure 4 | Inhibition of TRPC5 activity protects podocin and synaptopodin in human kidney organoids treated with PAN. (A,B) Immunostaining for podocin (A) and synaptopodin (B) in PBS, PAN and PAN + AC1903 treated kidney organoids. Scale bar $20 \mu \mathrm{m}$.

12. Welsh GI, Saleem MA. The podocyte cytoskeleton-key to a functioning glomerulus in health and disease. Nat Rev Nephrol. (2011) 8:14-21. doi: $10.1038 /$ nrneph.2011.151

13. Akilesh S, Suleiman H, Yu H, Stander MC, Lavin P, Gbadegesin R, et al. Arhgap24 inactivates Racl in mouse podocytes, and a mutant form is associated with familial focal segmental glomerulosclerosis. J Clin Invest. (2011) 121:4127-37. doi: 10.1172/JCI46458

14. Gee HY, Saisawat P, Ashraf S, Hurd TW, Vega-Warner V, Fang H, et al. ARHGDIA mutations cause nephrotic syndrome via defective RHO GTPase signaling. J Clin Invest. (2013) 123:3243-53. doi: 10.1172/JCI 69134

15. Yu H, Artomov M, Brahler S, Stander MC, Shamsan G, Sampson MG, et al. A role for genetic susceptibility in sporadic focal segmental glomerulosclerosis. J Clin Invest. (2016) 126:1603. doi: 10.1172/JCI 87342

16. Zhou Y, Castonguay P, Sidhom EH, Clark AR, Dvela-Levitt M, Kim S, et al. A small-molecule inhibitor of TRPC5 ion channels suppresses progressive kidney disease in animal models. Science. (2017) 358:13326. doi: $10.1126 /$ science.aal 4178

17. Montell C, Rubin GM. Molecular characterization of the Drosophila trp locus: a putative integral membrane protein required for phototransduction. Neuron. (1989) 2:1313-23. doi: 10.1016/0896-6273(89)90069-X

18. Montell C. Drosophila TRP channels. Pflugers Arch. (2005) 451:1928. doi: 10.1007/s00424-005-1426-2

19. Ilatovskaya DV, Palygin O, Chubinskiy-Nadezhdin V, Negulyaev YA, Ma R, Birnbaumer L, et al. Angiotensin II has acute effects on TRPC6 channels in podocytes of freshly isolated glomeruli. Kidney Int. (2014) 86:50614. doi: $10.1038 /$ ki.2014.71

20. Clapham DE. TRP channels as cellular sensors. Nature. (2003) 426:51724. doi: $10.1038 /$ nature 02196 
21. Pablo JL, Greka A. Charting a TRP to novel therapeutic destinations for kidney diseases. Trends Pharmacol Sci. (2019) 40:911-8. doi: 10.1016/j.tips.2019.10.001

22. Winn MP, Conlon PJ, Lynn KL, Farrington MK, Creazzo T, Hawkins AF, et al. A mutation in the TRPC6 cation channel causes familial focal segmental glomerulosclerosis. Science. (2005) 308:1801-4. doi: 10.1126/science.11 06215

23. Reiser J, Polu KR, Moller CC, Kenlan P, Altintas MM, Wei C, et al. TRPC6 is a glomerular slit diaphragm-associated channel required for normal renal function. Nat Genet. (2005) 37:739-44. doi: 10.1038/ng1592

24. Riehle M, Buscher AK, Gohlke BO, Kassmann M, Kolatsi-Joannou M, Brasen JH, et al. TRPC6 G757D loss-of-function mutation associates with FSGS. J Am Soc Nephrol. (2016) 27:2771-83. doi: 10.1681/ASN.2015030318

25. Cheng ZZ, Patari A, Aalto-Setala K, Novikov D, Schlondorff D, Holthofer H. Hypercholesterolemia is a prerequisite for puromycin inducible damage in mouse kidney. Kidney Int. (2003) 63:107-12. doi: 10.1046/j.1523-1755.2003.00726.x

26. Brideau G, Doucet A. Over-expression of adenosine deaminase in mouse podocytes does not reverse puromycin aminonucleoside resistance. BMC Nephrol. (2010) 11:15. doi: 10.1186/1471-2369-11-15

27. Moller CC, Wei C, Altintas MM, Li J, Greka A, Ohse T, et al. Induction of TRPC6 channel in acquired forms of proteinuric kidney disease. J Am Soc Nephrol. (2007) 18:29-36. doi: 10.1681/ASN.2006091010

28. Sun X, Fang Z, Zhu Z, Yang X, He F, Zhang C. Effect of downregulation of TRPC6 on the puromycin aminonucleoside-induced apoptosis of mouse podocytes. J Huazhong Univ Sci Technolog Med Sci. (2009) 29:41722. doi: 10.1007/s11596-009-0405-9

29. Wang Z, Wei X, Zhang Y, Ma X, Li B, Zhang S, et al. NADPH oxidasederived ROS contributes to upregulation of TRPC6 expression in puromycin aminonucleoside-induced podocyte injury. Cell Physiol Biochem. (2009) 24:619-26. doi: 10.1159/000257517

30. Sun X, Chu Y, Zhang C, Du X, He F, Chen S, et al. Effect of TRPC6 knockdown on puromycin aminonucleoside-induced podocyte injury. J Huazhong Univ Sci Technolog Med Sci. (2012) 32:340-5. doi: 10.1007/s11596-012-0059-x

31. Kim EY, Yazdizadeh Shotorbani P, Dryer SE. Trpc6 inactivation confers protection in a model of severe nephrosis in rats. J Mol Med. (2018) 96:63144. doi: 10.1007/s00109-018-1648-3

32. Ciampi O, Iacone R, Longaretti L, Benedetti V, Graf M, Magnone $\mathrm{MC}$, et al. Generation of functional podocytes from human induced pluripotent stem cells. Stem Cell Res. (2016) 17:130-9. doi: 10.1016/j.scr.2016. 06.001

33. Saleem MA, O'hare MJ, Reiser J, Coward RJ, Inward CD, Farren T, et al. A conditionally immortalized human podocyte cell line demonstrating nephrin and podocin expression. J Am Soc Nephrol. (2002) 13:6308. doi: 10.1681/ASN.V133630

34. Shankland SJ, Pippin JW, Reiser J, Mundel P. Podocytes in culture: past, present, and future. Kidney Int. (2007) 72:26-36. doi: 10.1038/sj.ki.50 02291

35. Schaldecker T, Kim S, Tarabanis C, Tian D, Hakroush S, Castonguay P, et al. Inhibition of the TRPC5 ion channel protects the kidney filter. J Clin Invest. (2013) 123:5298-309. doi: 10.1172/JCI71165
36. Takasato M, Er PX, Chiu HS, Maier B, Baillie GJ, Ferguson C, et al. Kidney organoids from human iPS cells contain multiple lineages and model human nephrogenesis. Nature. (2015) 526:564-8. doi: 10.1038/nature15695

37. Takasato M, Er PX, Chiu HS, Little MH. Generation of kidney organoids from human pluripotent stem cells. Nat Protoc. (2016) 11:1681-92. doi: 10.1038/nprot.2016.098

38. Subramanian A, Sidhom EH, Emani M, Vernon K, Sahakian N, Zhou Y, et al. Single cell census of human kidney organoids shows reproducibility and diminished off-target cells after transplantation. Nat Commun. (2019) 10:5462. doi: 10.1038/s41467-019-13382-0

39. Yu H, Suleiman H, Kim AH, Miner JH, Dani A, Shaw AS, et al. Racl activation in podocytes induces rapid foot process effacement and proteinuria. Mol Cell Biol. (2013) 33:4755-64. doi: 10.1128/MCB.00730-13

40. Hall G, Spurney RF. Losing their footing: Rac1 signaling causes podocyte detachment and FSGS. Kidney Int. (2017) 92:283-5. doi: 10.1016/j.kint.2017.03.045

41. Robins R, Baldwin C, Aoudjit L, Cote JF, Gupta IR, Takano T. Racl activation in podocytes induces the spectrum of nephrotic syndrome. Kidney Int. (2017) 92:349-64. doi: 10.1016/j.kint.2017.03.010

42. Lv Z, Hu M, Fan M, Li X, Lin J, Zhen J, et al. Podocyte-specific Racl deficiency ameliorates podocyte damage and proteinuria in STZ-induced diabetic nephropathy in mice. Cell Death Dis. (2018) 9:342. doi: 10.1038/s41419-018-0353-Z

43. Yu M, Ledeboer MW, Daniels M, Malojcic G, Tibbitts TT, Coeffet-Le Gal $\mathrm{M}$, et al. Discovery of a potent and selective TRPC5 inhibitor, efficacious in a focal segmental glomerulosclerosis model. ACS Med Chem Lett. (2019) 10:1579-85. doi: 10.1021/acsmedchemlett.9b00430

Conflict of Interest: AG has a financial interest in Goldfinch Biopharma, which was reviewed and is managed by Brigham and Women's Hospital/MassGeneralBrigham (MGB) and the Broad Institute of MIT and Harvard in accordance with their conflict of interest policies.

The remaining authors declare that the research was conducted in the absence of any commercial or financial relationships that could be construed as a potential conflict of interest.

Publisher's Note: All claims expressed in this article are solely those of the authors and do not necessarily represent those of their affiliated organizations, or those of the publisher, the editors and the reviewers. Any product that may be evaluated in this article, or claim that may be made by its manufacturer, is not guaranteed or endorsed by the publisher.

Copyright (C) 2021 Zhou, Kim, Pablo, Zhang, Jung, Xiao, Bazua-Valenti, Emani, Hopkins, Weins and Greka. This is an open-access article distributed under the terms of the Creative Commons Attribution License (CC BY). The use, distribution or reproduction in other forums is permitted, provided the original author(s) and the copyright owner(s) are credited and that the original publication in this journal is cited, in accordance with accepted academic practice. No use, distribution or reproduction is permitted which does not comply with these terms. 